\title{
Effect of Curcumin, Mixture of Curcumin and Piperine and Curcum (Turmeric) on Lipid Profile of Normal and Hyperlipidemic Rats
}

\author{
GHADA, Z. A. Soliman \\ Lecturer of Biochemistry, Biochemistry Department, National \\ Nutrition Institute, Cairo
}

\begin{abstract}
Curcumin is a polyphenolic, yellow pigment obtained from rhizomes of Curcuma longa (curcum), used as a spice and food colouring. The extracts have several pharmacological effects. We evaluated the effect of curcum, curcumin, and mixture of curcumin and piperine on plasma lipids in normal and hypercholesterolemic rats. A total of 270 rats, divided into 27 groups, were used. G1, G1 ${ }^{1}$ : control, G2-G11: normal rats fed control diet supplemented with different levels of curcumin and curcum (G2-G6: $0.1 \%, 0.25 \%, 0.5 \%, 1.0 \%, 2.0 \%$ respectively, G7-G11: $1.67 \%, 4.167 \%, 8.34 \%, 16.67 \%$, and 33.34). G12-G26: at first fed control diet supplemented with $2 \%$ cholesterol then G13-17, 21-25 fed a control diet supplemented with different levels of curcumin, and curcum [the same levels as G2-G11; G18-20 fed control diet supplemented with mixture of curcumin $(0.1,0.25,0.5 \%)$ and piperine $(20 \mathrm{mg} / \mathrm{kg} \mathrm{BW})], \mathrm{G} 12$ was sacrificed before addition of studied materials, G26 were fed control diet. Lipid profile, triacylglycerol and phospholipids of plasma and organs as liver and heart were measured. Serum cholesterol (total, LDL-C, VLDL-C), triacylglycerol and phospholipids contents were elevated in cholesterol-fed rats, while HDL-C were decreased. Curcum, curcumin have hypocholesterolemic effect on both normal and hypercholesterolemic rats being more effective in hypercholesterolemic rats. Curcumin reduces cholesterol by interfering with intestinal cholesterol uptake, increasing the conversion of cholesterol into bile acids and increasing the excretion of bile acids. Using curcumin+piperine is better than using curcumin alone. All doses had the same effect, but using the lower level $(0.5 \%)$ is better than using $2.0 \%$ level. Liver cholesterol, triacylglycerol and phospholipids contents and cardiac cholesterol were elevated in hypercholesterolemic conditions. Dietary curcumin showed a distinct tendency to counter these changes. Piperine was added to curcumin to enhance its bioavailabilty through increasing curcumin absorption and reducing its metabolism in liver. The use of curcum, curcumin, and mixture of curcumin and piperine may be useful in the management of cardiovascular disease. Using the lower level $(0.5 \%)$ is better than using $2.0 \%$. Piperine enhances the bioavailabilty of curcumin.
\end{abstract}

Keywords: Curcuma longa L.; Atherosclerosis; Antioxidant, Cholesterol, HDL-C, LDL-C, VLDL-C, Triacylglycerol, Phospholipids, Curcumin, Piperine, Hypercholesterolemia, Liver, Heart

\section{Introduction}

Curcuma longa L. plant (known as curcum hence the name Curcuma, turmeric) is a member of the Curcuma botanical group, which is part of the ginger family of herbs, Zingiberaceae. It is widely cultivated in tropical regions of Asian countries including India. The root and rhizome (underground stem) of the Curcuma longa $L$. plant is crushed and powdered into ground Turmeric. Ground Turmeric is used worldwide as the main ingredient in curry, the spice, and as a source for curcumin or curcuminoids. Curcum is a well-known spice and food colorant commonly consumed in different parts of the world. Recently, much attention has been focused on the biological and medicinal properties of curcumin. Curcumin, a group of polyp- 
henolic plant pigments, is responsible for turmeric's characteristic canary yellow color. Curcuminoids include mostly curcumin, demethoxycurcumin and bisdemethoxycurcumin. Turmeric powder also is used for medicinal purpose and in Ayurvedic medicine (Ivan 1999 and WHO 2002). Turmeric is on the FDA's GRAS (Generally Recognized as safe) list. No $\mathrm{LD}_{50}$ has been discovered for curcumin (Bratman and Girman 2003). Cheng et al. 2001 found that curcumin is not toxic in oral human doses up to $8000 \mathrm{mg} /$ day for 3 months. Curcumin has a wide range of therapeutic actions as the ability to halt or prevent certain types of cancer (Aggarwal et al. 2003, Bharti et al. 2003 and Chan et al. 2003), anti-inflammation (Ramsewak et al. 2000); improve cardiovascular health (Ramirez-Tortosa et al. 1999, Quiles et al. 2002 and Mesa et al. 2003); prevent cataracts (Suryanarayana et al. 2003); antimicrobial, anti-fungi (Saleheen et al. 2002); remarkable antioxidant reagent (RamirezBoscá et al. 1995, Sreejayan and Rao 1996 and Phan et al. 2001). Curcum (Curcuma longa), curcumin has hypocholesterolemic effect on hypercholesterolemic rats. Curcumin (turmeric) or curcum decrease (suppress) the raised level of liver cholesterol of cholesterol-fed rats (Reddy and Lokesh 1994). The hypocholesterolemic effect of curcum may be due the presence of curcumin and/or curcumonoinds (mixture of curcumin and relative compounds). Curcumin reduces cholesterol by interfering with intestinal cholesterol uptake, increasing the conversion of cholesterol into bile acids, and increasing the excretion of bile acids Ravindranath and Chandrasekhara 1980 and 1981 and Ammon and Wahl 1991 indicated that pharmacokinetic properties of curcumin reveal that curcumin when given orally is less active due to its poor absorption by the gastrointestinal tract and its glucuronidation in the liver. Addition of piperine (extract of black pepper, Piper nigrum L.) to curcumin enhances its bioavailabilty through increasing curcumin absorption and reducing its metabolism in liver (Shoba et al. 1997).

\section{Materials and Methods}

\section{A-Experimental Animals}

Two hundred and seventy male albino, Sprague-Dawley (local strain), rats were housed individually in suspended wire-mesh cages on two steps. All rats were initially fed basal (control) diet for 10 days before starting the experiment. Rats were then divided into 27 groups, each of 10 rats. Their mean body weight was $164.0 \mathrm{~g}$ ranging between 151-179 g. Water and diets were given ad libitum. The first experiment lasted for 7 weeks; the second experiment lasted for another 7 weeks.

\section{B-Animal Grouping}

Group 1 (control): fed on control diet. From the $2^{\text {nd }}$ to the $6^{\text {th }}$ group animals were fed on control diet supplemented with different levels of curcumin $(0.1 \%, 0.25 \%$, $0.5 \%, 1.0 \%$, and $2.0 \%$ respectively). From the $7^{\text {th }}$ to the $11^{\text {th }}$ group animals were fed on control diet supplemented with different levels of curcum that contain the same concentration of curcumin that were given to groups $2-6(1.67 \%, 4.167 \%, 8.34 \%$, $16.67 \%$, and $33.34 \%$ respectively). From the $12^{\text {th }}$ to the $26^{\text {th }}$ group animals were fed on control diet supplemented with $2 \%$ cholesterol in order to make a hyperlipidemic rats. The $27^{\text {th }}$ (or $1^{1}$ ) group was fed a control diet to act as a control for the second experiment (treatment of the hyperlipidemic rats with curcum, curcumin and a mixture of curcumin and piperine). Curcum and curcumin were dissolved in oil. The addition of curcum, curcumin and cholesterol was at the expense of cornstarch. The diets were prepared fresh every week and kept at $4^{\circ} \mathrm{C}$. The control diet was prepared according to National Research Council, NRC, (1978) and Reeves et al. (1993).

At the end of first 7 weeks of the experiment, the rats (from group 1-11 and 26) were fasted overnight, lightly anaesthetized with diethyl ether to collect blood samples into dry clean centrifuge tube for biochemical analysis of Cholesterol (total, HDL, LDL and VLDL), 
triacylglycerol (TG) and total phospholipids $(\mathrm{PhL})$.

Liver and heart were removed immediately after dissection and rinsed with saline, dried between two filter papers and weighed. Lipoprotein (lipid pattern, total cholesterol, total TG and total phospholipids) was also measured in liver. Total cholesterol was also measured in heart.

At the second experiment part, Groups 12-25, 26 became hyperlipidemic, group $27\left(1^{1}\right)$ was used as normal control rats. In Group 26, analytical parameters were used as the initial level of lipid profile of hypercholesterolemic rats. Group 12 were kept on control diet with no supplementation during the other 7 weeks where it was considered as hypercholesterolemic control group of rats. From the $13^{\text {th }}$ to the $17^{\text {th }}$ group animals were fed on control diet supplemented with different levels of curcumin $(0.1 \%, 0.25 \%, 0.5 \%$, $1.0 \%$, and $2.0 \%$ respectively). From the $18^{\text {th }}$ to the $20^{\text {th }}$ group animals were fed on control diet supplemented with different levels of curcumin $(0.1 \%, 0.25 \%, 0.5 \%$ respectively) and piperine $(20 \mathrm{mg} / \mathrm{kg} \mathrm{BW})$ for the 3 groups. From the $21^{\text {st }}$ to the $25^{\text {th }}$ group animals were fed on control diet supplemented with different levels of curcumin that contain the same concentration of curcumin that were given to groups $13-17(1.67 \%, 4.167 \%, 8.34 \%$, $16.67 \%$, and $33.34 \%$ respectively).

At the end of last 7 weeks of the experiment, the rats were fasted overnight, lightly anaesthetized and sacrificed. Then biochemical analysis of blood, heart, liver was done as previous.

\section{C-Measured Parameters}

Initial body weight (IBW), final body weight (FBW) and body weight gain (BWG). Absolute organs weight (AOW: g upon autopsy) and relative organs weight (ROW) and atherogenic indices were calculated.

\section{D-Chemical Analysis}

The contents were determined using suitable kits reagents. Total cholesterol, TC, (Bio Mérieux kits -Richmond 1973 and
Allain et al. 1974), total triacylglycerol, TG, (Bicon kits -Bucolo and David 1973), total phospholipids (Connerty et al. 1961), serum HDL (Bio Mérieux kits -Burstein et al. 1970 and Lopes Virella et al. 1977), serum LDL (Bio Mérieux kits -Friedewald et al. 1972, Levy et al. 1981 and Fruchart 1982). VLDL-C was determined by using the following equation: VLDL-C=total cholesterol - (HDL-C + LDL-C).

Lipids were extracted from the liver and heart using Folch et al. (1957) and Carr et al. (1993) methods, then TC, TG, Tphl content was determined in them.

\section{E-Chemicals used}

Curcumin and piperine were purchased from Sigma. Curcumin is natural yellow, 1,7-bis (4-hydroxy-3-methylphenyl) 1-6 heptadiene-3-dione. Its Molecular weight is $\mathrm{C}_{21} \mathrm{H}_{20} \mathrm{O}_{6}$. Piperine molecular weight is $\mathrm{C}_{17} \mathrm{H}_{19} \mathrm{NO}_{3}$.

\section{Statistical Analysis}

Data were expressed as Mean \pm SE and assessed by paired t-test (Avram 1964) and Duncan test (Steel and Torrie 1960).

\section{Results}

Table (1-a) revealed that body weight gain (BWG) of curcumin- or curcum-fed normal rats was similar with no significant differences between them. The treated groups showed slightly non significant decrease in their BWG [-1.46 (G 2): -9.02 (G 10)] than control.

Table (1-b) revealed that FBW of cholesterol fed rats was significantly higher than that of their respective control group or their respective initial groups $(244.19 \pm 0.59$ vs $227.0 \pm 2.51$ and $164.55 \pm 1.91$ respectively). Body weight gain was significantly higher than control group especially group 12 where they reached $34.35 \%$.

Table (1-c) revealed that all groups (G: 13-25) showed no significant change than their respective hypercholesterolemic control groups (G 12), although they showed slightly higher weights. Also body weight showed a tendency to increase with increasing concentration of the added curcumin, curcumin+piperine and curcum. 
Body weight gain was significantly lower than normal control group.

Regarding organs weight, Table (2-a) showed that relative liver and heart weight of curcumin- or curcum-fed normal rats showed no significant differences when compared with the control group with tendency of heart weight to be slightly lower than control. Relative liver and heart weight of cholesterol-fed group (G 26; Table 2-b) showed a significant increase when compared with the control group. Relative liver weight of all treated hypercholesterolemic groups (G: 13-25) showed a highly significant decrease when compared with normal control group or compared with initial hypercholesterolemic group (G 26). Also no significant differences for RLW were found when compared with the untreated hypercholesterolemic control group (G 12), which is quite different for relative heart weight. All groups (G: 13-25) showed no significant change when compared with normal controls $\left(\mathrm{G} 1^{1}\right)$ and a significant decrease was found on comparing with G 12 . Relative heart weight of curcumin-fed (G: 14-17); curcumin+piperine-fed ( $\mathrm{G} 20$ ) and curcum-fed (G:22-25) of hypercholesterolemic rats was significantly lower than initial hypercholesterolemic group (G 26).

Table (3-a, b) revealed that serum cholesterol (total, HDL-C, LDL, VLDL) triacylglycerol, and phospholipids levels of $0.1 \%$ and $0.25 \%$ curcumin- or curcum- fed normal groups (2-3 and 7-8) showed no significant differences when compared with the control, but serum cholesterol (total, LDL, VLDL) of the remaining groups (G: 4-6 and 9-11) of curcumin- or curcum- fed groups was significantly lower than control group. Also serum HDL-C of groups (G: 46 and 9-11) of curcumin- and/or curcumfed groups was significantly higher than control group. Most of cholesterol decreases occured in LDL and VLDL fraction $(\approx 20 \%$ each). Triacylglycerol and phospholipids levels of low level of curcumin- or curcum- fed groups (G: 2-4; 7-9) showed no significant differences when compared with the control group, while high level of curcumin- or curcumfed groups (G: 11; 5-6) showed a significant decrease when compared with the control group except G 10 which showed no significant change.

Table $(4-a, b, c)$ revealed that serum cholesterol (total, LDL, VLDL); triacylglycerol and phospholipids levels of cholesterol-fed groups were significantly higher than control group, while HDL-C was significantly lower than control group. The increase reached $109.77 \%, 295.39 \%$; $281.64 \%, 96.76 \%$; and $81.85 \%$ respectively, while the decrease in serum HDL-C reached $46.01 \%$. The present study also showed that approximately $53.75 \%$ of normal control rats' serum cholesterol was present in HDL form and relatively little in LDL and VLDL (32.04 and14.19\%), while in hypercholesterolemic rat it was quite different, approximately 13.83 in HDL, and 60.37 and 25.8 in LDL and VLDL. Table (4-a) also revealed that serum cholesterol (total, LDL, VLDL); triacylglycerol and phospholipids levels of untreated hyperlipidemic control groups (G 12) were significantly higher than control group, while HDL-C was significantly lower than control group but the opposite was true when they are compared with the initial level of cholesterol-fed group.

Tables $(4-a, b, c)$ revealed that serum cholesterol (total, LDL, VLDL); triacylglycerol and phospholipids levels of groups (13; 18; 21, low level) behave like G 12 , then the remaining groups $(14-17 ; 19-20$; 22-25) were significantly decrease in cholesterol (total, LDL, VLDL); triacylglycerol and phospholipids, while their HDL-C was significantly increasing. This is true for all groups except groups $(17,20$, 25) where the levels became close to normal where cholesterol (total, HDL-C), triacylglycerol and phospholipids showed no significant change when compared with the normal group $\left(1^{1}\right)$ but LDL-C and VLDL-C of G 17 showed significant change (increase). Data of table (4-b) revealed that curcumin+piperine is much better than curcumin alone as hypocholesterolemic agents.

Regarding atherogenic indices, data of table (3-a and b) reveal that groups 2-3 and 7-8 (normal rats) showed no significant change for all indicated atherogenic indices 
when compared with the normal control. G: 4-6 and 9-11 showed a significant decrease in cholesterol/HDL-C, LDL-C/HDL-C. Also no significant change was observed when comparing between each group and its match $(7,8,9,10,11$ vs $2,3,4,5,6$ respectively).

Data of cholesterol/HDL-C and LDLC/HDL-C showed significantly higher levels in cholesterol-fed group (G 26) than control one.

Group 12 (untreated hypercholesterolemic control) behaved like G 26 when compared with the normal control, but the opposite is true when comparing it with $\mathrm{G}$ 26. Groups 13-25 showed significant changes when compared with $\mathrm{G} 1^{1} ; 26,12$. The atherogenic indices showed a tendency to either increase or decrease with increasing concentration of the added curcumin, curcumin+piperine and curcum when compared with $\mathrm{G} 1^{1} ; 26,12$ (Table 4$\mathrm{a}, \mathrm{b}$ and c) i.e. cholesterol/HDL-C, LDLC/HDL-C of G 13 (lower level) showed significant increase than normal control group and G 12 (untreated hypercholesterolemic control group) and showed a significant decrease when compared with $G$ 26 (initial hypercholesterolemic control group). The levels decrease with increasing concentration of the added test material (revert into significant decrease) till it became significantly higher than $\mathrm{G} 1^{1}$, and significantly lower than G 26 and 12 .

Table (5-a) revealed that hepatic cholesterol content of all groups (3-6, 8-11) of normal curcumin- or curcum- fed groups showed a significantly lower values when compared with the control except group (2, 7) which showed non significant change, while liver triacylglycerol content showed no significant change for all groups except groups (5,6 and 10,11, high level fed) which showed a significant decrease when compared with the normal group. Hepatic phospholipids and cardiac cholesterol content showed non significant change.
Table (5-b) showed significantly higher levels of hepatic cholesterol, triacylglycerol and phospholipids concentrations; and cardiac cholesterol concentration in cholesterol-fed group (G 26) or the untreated hypercholesterolemic control group (G 12) than control group.

The untreated hypercholesterolemic control group (G 12) showed significantly lower levels of hepatic cholesterol, triacylglycerol and phospholipids concentrations; and a non significant change of cardiac cholesterol than cholesterol-fed group ( $\mathrm{G}$ 26).

Data of table (5-b) showed significantly lower levels of hepatic cholesterol, triacylglycerol concentrations; and cardiac cholesterol concentration in curcumin-fed (G: 13-17), curcumin+ piperine-fed (G: 1820), curcum-fed (G: 21-25) groups when compared with control $\left(\mathrm{G} 1^{1}\right)$, or with untreated hypercholesterolemic control group (G 12) or with control hyperlipidemic group (G 26) except $\mathrm{G} 18$ and 22 when compared with G 12 where no significant change was observed.

Hepatic phospholipids contents of all groups showed no significant change when compared with $G 12$ or $G 1^{1}$ except $G$ 13 and $G$ 21, which showed significant increase when compared with G $1^{1}$. Hepatic phospholipids contents of all groups showed a significant decrease when compared with G 26 except G 13 and G 21, which showed non significant decrease.

Cardiac cholesterol contents of groups (G: $15-17 ; 19-20 ; 23-25$ ) showed no significant change when compared with $G$ $1^{1}$ except G $13-14 ; 18 ; 21-22$ which showed significant increase when compared with $\mathrm{G}$ $1^{1}$. Cardiac cholesterol contents of all groups showed no significant change when compared with G 12 or G26 except G 16$17,20,23-25$, which showed a significant decrease when compared with G 26 and G 17,25 , which showed a significant decrease when compared with G 12 . 
Effect of Curcumin, Mixture of Curcumin and..........

Table (1-a): Body weight gain, g, (BWG) of normal rats supplemented with different of curcum or curcumin

\begin{tabular}{|l|l|l|l|l|l|l|l|l|l|l|l|}
\hline & G1: Control & G 2:Curn 1 & G 3:Curn 2 & G 4:Curn 3 & G 5:Curn 4 & G 6:Curn 5 & G 7: Cur 1 & G 8:Cur 2 & G 9:Cur 3 & G 10:Cur 4 & G 11:Cur 5 \\
\hline${\mathrm{M} \pm \mathrm{SE}^{*}}$ & $62.45 \pm 2.03$ & $61.55 \pm 4.18$ & $59.18 \pm 2.46$ & $58.45 \pm 5.02$ & $58.18 \pm 2.15$ & $57.00 \pm 1.69$ & $60.18 \pm 2.47$ & $58.91 \pm 1.58$ & $57.64 \pm 1.86$ & $56.82 \pm 1.94$ & $57.00 \pm 1.76$ \\
\hline
\end{tabular}

Table (1-b): Body weight gain, g, (BWG) of hypercholesterolemic rats

\begin{tabular}{|c|c|c|c|c|c|c|c|c|c|c|c|c|c|c|c|c|}
\hline & G 1 & G 12 & G 13 & G 14 & G 15 & G 16 & G 17 & G 18 & G 19 & G 20 & G 21 & G 22 & G 23 & G 24 & G 25 & G 26 \\
\hline \begin{tabular}{|c|} 
Additi \\
on
\end{tabular} & \begin{tabular}{|c|}
$\begin{array}{c}\text { Control } \\
\text { Non }\end{array}$ \\
\end{tabular} & \multicolumn{15}{|c|}{ Cholesterol treated (3\%Cholesterol) } \\
\hline \begin{tabular}{c||}
$M \pm S$ \\
$E$
\end{tabular} & $\begin{array}{c}62.45 \pm 2 . \\
03\end{array}$ & \begin{tabular}{|c|}
$83.91 \pm 1$ \\
59
\end{tabular} & \begin{tabular}{|c|}
$80.36 \pm 3$ \\
19
\end{tabular} & $\begin{array}{c}80.18 \pm 3 \\
65\end{array}$ & $\begin{array}{c}80.36 \pm 3 \\
60\end{array}$ & $\begin{array}{c}79.64 \pm 2 . \\
75\end{array}$ & \begin{tabular}{|c|}
$76.91 \pm 2$ \\
55
\end{tabular} & $\begin{array}{c}80.64 \pm 2 . \\
43\end{array}$ & \begin{tabular}{|c|}
$80.55 \pm 1$ \\
38
\end{tabular} & $\begin{array}{c}78.45 \pm 1 \\
65\end{array}$ & $\begin{array}{c}81.82 \pm 1 \\
83\end{array}$ & $\begin{array}{c}79.45 \pm 2 . \\
21\end{array}$ & \begin{tabular}{|c|}
$79.18 \pm 3$ \\
68
\end{tabular} & $\begin{array}{c}80.27 \pm 2 \\
30\end{array}$ & \begin{tabular}{|c|}
$79.00 \pm 3$ \\
26
\end{tabular} & $\begin{array}{l}78.45 \\
\pm 2.42 \\
\end{array}$ \\
\hline $\mathrm{P} \leq$ & & 0.0001 & 0.0001 & 0.0004 & 0.0003 & 0.0001 & 0.0003 & 0.0001 & 0.0001 & 0.0001 & 0.0001 & 0.0001 & 0.0007 & 0.0001 & 0.0003 & 0.0001 \\
\hline
\end{tabular}

Table (1-c): Body weight gain, g, (BWG) of hypercholesterolemic rats supplemented with different levels of curcum or curcumin or curcumin +piperine

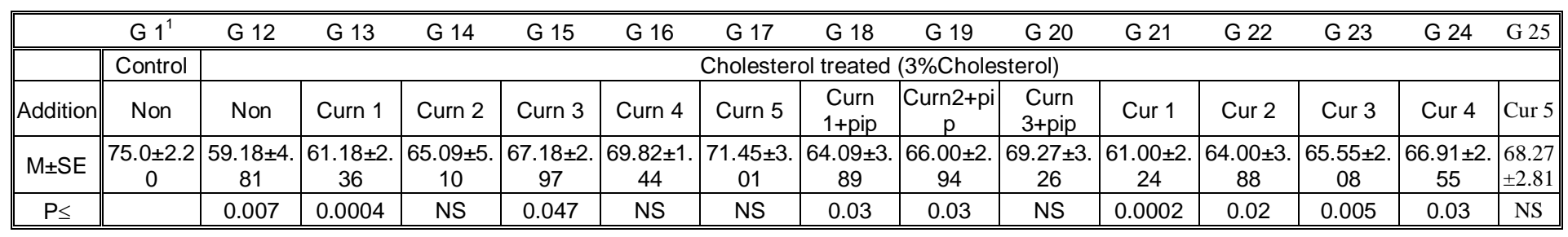

Table (2-a): Relative weight [(organ weight/FBW)*100] of liver and heart of normal rats fed different levels of curcum or curcumin. Results are expressed as $\mathrm{M} \pm \mathrm{SE}$

\begin{tabular}{|c|c|c|c|c|c|c|c|c|c|c|c|}
\hline & G1: Control & G 2:Curn 1 & G 3:Curn 2 & G 4:Curn 3 & G 5:Curn 4 & G 6:Curn 5 & G 7: Cur 1 & G 8:Cur 2 & G 9:Cur 3 & G 10:Cur 4 & G 11:Cur 5 \\
\hline $\mathrm{RLW}^{*}$ & $3.79 \pm 0.05$ & $3.82 \pm 0.03$ & $3.87 \pm 0.05$ & $3.86 \pm 0.04$ & $3.90 \pm 0.04$ & $3.90 \pm 0.03$ & $3.85 \pm 0.06$ & $3.87 \pm 0.03$ & $3.91 \pm 0.05$ & $3.91 \pm 0.03$ & $3.91 \pm 0.03$ \\
\hline
\end{tabular}

Table (2-b): Relative weight [(organ weight/FBW $)^{* 100]}$ of liver and heart of hypercholesterolemic rats fed different levels of curcum or curcumin or curcumin+piperine

\begin{tabular}{|c|c|c|c|c|c|c|c|c|c|c|c|c|c|c|c|c|}
\hline & $\mathrm{G} 1^{1}$ & G 26 & G 12 & G 13 & G 14 & G 15 & G 16 & G 17 & G 18 & G 19 & G 20 & G 21 & G 22 & G 23 & G 24 & G 25 \\
\hline & Cont & \multicolumn{15}{|c|}{ Cholesterol treated(3\%Cholesterol) } \\
\hline Addition & Non & Non (I) & Non (II) & Curn 1 & Curn 2 & Curn 3 & Curn 4 & Curn 5 & $\begin{array}{l}\text { Curn } \\
1+\text { pip }\end{array}$ & \begin{tabular}{|c|}
$\begin{array}{c}\text { Curn2+ } \\
\text { pip }\end{array}$ \\
\end{tabular} & $\begin{array}{c}\text { Curn } \\
3+\text { pip }\end{array}$ & Cur 1 & Cur 2 & Cur 3 & Cur 4 & Cur 5 \\
\hline RLW & \begin{tabular}{|c|}
$3.75 \pm 0$. \\
05
\end{tabular} & $\begin{array}{c}4.09 \pm 0 . \\
10\end{array}$ & \begin{tabular}{|c|}
$2.99 \pm 0$ \\
$06^{1,2}$
\end{tabular} & \begin{tabular}{|c|}
$3.12 \pm 0$ \\
$09^{1,2}$ \\
\end{tabular} & \begin{tabular}{|c|}
$3.07 \pm 0$. \\
05 \\
1,2 \\
\end{tabular} & \begin{tabular}{|c|}
$3.02 \pm 0$ \\
$07^{1,2}$
\end{tabular} & \begin{tabular}{|c|}
$2.98 \pm 0$ \\
$08^{1,2}$
\end{tabular} & \begin{tabular}{|c|}
$2.93 \pm 0$ \\
$06^{1,2}$
\end{tabular} & \begin{tabular}{|c|}
$3.01 \frac{1}{1,0}$. \\
$06^{1,2}$ \\
\end{tabular} & \begin{tabular}{|c|}
$3.00 \pm 0$ \\
$111^{1,2}$
\end{tabular} & \begin{tabular}{|c|}
$2.99 \pm 00$ \\
$08^{1,2}$ \\
\end{tabular} & \begin{tabular}{|c|}
$3.03 \div 0$ \\
$10^{1,2}$ \\
\end{tabular} & $\mid \begin{array}{c}3.03 \pm 0 \\
09^{1,2}\end{array}$ & $\begin{array}{c}2.99 \pm 0 \\
05^{1,2}\end{array}$ & $\begin{array}{c}2.98 \pm 0 \\
09^{1,2}\end{array}$ & $\begin{array}{c}2.950 .1 \\
0^{1,2}\end{array}$ \\
\hline RHW & $\mid$\begin{tabular}{|l|}
0.3375 \\
\pm 0.005
\end{tabular} & $\begin{array}{l}0.3685 \\
\pm 0.01^{1}\end{array}$ & $\begin{array}{l}0.382 \pm \\
0.008^{1}\end{array}$ & \begin{tabular}{|c|}
0.3495 \\
\pm 0.008 \\
3
\end{tabular} & \begin{tabular}{|c|}
0.3453 \\
$\pm 0.006^{2}$ \\
, 3
\end{tabular} & $\begin{array}{l}0.344 \pm 0 \\
.007^{2,3}\end{array}$ & $\begin{array}{c}0.3407 \\
\pm 0.01 \\
2,3\end{array}$ & $\begin{array}{c}0.3375 \\
\pm 0.006^{2} \\
, 3\end{array}$ & $\begin{array}{c}0.35 \pm 0 \\
006^{3}\end{array}$ & $\begin{array}{l}0.3430 \\
\pm 0.01^{3}\end{array}$ & $\begin{array}{l}0.339 \pm \\
0.01^{2,3}\end{array}$ & $\begin{array}{l}0.350 \pm \\
0.01^{\frac{3}{3}}\end{array}$ & $\begin{array}{l}0.343 \pm \\
0.008^{2,3}\end{array}$ & $\begin{array}{c}0.3412 \\
\pm 0.008 \\
2,3\end{array}$ & $\left|\begin{array}{c}0.3401 \pm \\
0.01^{2,3}\end{array}\right|$ & $0.3374 \pm$ \\
\hline
\end{tabular}

The superscripts refer to G no., which are significant with (1: G $1^{1} ; 2$ : G 26; 3: G 12;) 
GHADA, Z. A. Soliman

Table (3-a): Effect of feeding different levels of curcum on lipid profile of normal rats (mg \%)

\begin{tabular}{|c|c|c|c|c|c|c|c|c|c|c|}
\hline & & & Cholesterol & HDL-C & LDL-C & VLDL-C & $\begin{array}{l}\text { Triacyl- } \\
\text { glycerol }\end{array}$ & $\begin{array}{l}\text { Phospho- } \\
\text { Lipids }\end{array}$ & $\frac{\text { Cholesterol }}{\text { HDL-C }}$ & $\frac{\mathrm{LDL}-\mathrm{C}}{\mathrm{HDL}-\mathrm{C}}$ \\
\hline 1 & Control I & $\mathrm{M} \pm \mathrm{SE}$ & $83.39 \pm 1.19$ & $44.84 \pm 0.87$ & $26.71 \pm 0.72$ & $11.82 \pm 0.36$ & $102.47 \pm 1.19$ & $541.72 \pm 3.48$ & $1.86 \pm 0.015$ & $0.60 \pm 0.02$ \\
\hline \multirow{2}{*}{2} & \multirow{2}{*}{ Curcum } & $\mathrm{M} \pm \mathrm{SE}$ & $81.16 \pm 2.14$ & $42.77 \pm 0.91$ & $26.59 \pm 1.28$ & $11.8 \pm 0.35$ & $100.21 \pm 1.86$ & $536.47 \pm 6.29$ & $1.90 \pm 0.027$ & $0.62 \pm 0.025$ \\
\hline & & $\mathrm{P} \leq$ & NS & NS & NS & NS & NS & NS & NS & NS \\
\hline \multirow{2}{*}{3} & \multirow{2}{*}{ Curcum } & $\mathrm{M} \pm \mathrm{SE}$ & $79.91 \pm 1.16$ & $42.3 \pm 0.93$ & $25.88 \pm 1.15$ & $11.73 \pm 0.47$ & $99.18 \pm 1.31$ & $532.47 \pm 5.29$ & $1.89 \pm 0.032$ & $0.61 \pm 0.027$ \\
\hline & & $P \leq$ & NS & NS & NS & NS & NS & NS & NS & NS \\
\hline \multirow{2}{*}{4} & \multirow{2}{*}{ Curcum } & $\mathrm{M} \pm \mathrm{SE}$ & $78.88 \pm 1.25$ & $47.24 \pm 0.54$ & $21.79 \pm 1.04$ & $9.85 \pm 0.19$ & $98.82 \pm 1.70$ & $533.82 \pm 5.92$ & $1.67 \pm 0.02$ & $0.46 \pm 0.02$ \\
\hline & & $\mathrm{P} \leq$ & 0.02 & 0.03 & 0.001 & 0.0001 & NS & NS & 0.0001 & 0.0001 \\
\hline \multirow{2}{*}{5} & \multirow{2}{*}{ Curcum } & $\mathrm{M} \pm \mathrm{SE}$ & $77.86 \pm 1.04$ & $47.13 \pm 0.52$ & $21.59 \pm 0.58$ & $9.14 \pm 0.29$ & $97.53 \pm 1.41$ & $528.69 \pm 3.56$ & $1.65 \pm 0.01$ & $0.46 \pm 0.01$ \\
\hline & & $\mathrm{P} \leq$ & 0.003 & 0.036 & 0.0001 & 0.0001 & 0.015 & 0.02 & 0.0001 & 0.0001 \\
\hline \multirow{2}{*}{6} & \multirow{2}{*}{ Curcum } & $\mathrm{M} \pm \mathrm{SE}$ & $77.67 \pm 0.99$ & $47.11 \pm 0.51$ & $21.47 \pm 0.57$ & $9.09 \pm 0.27$ & $95.22 \pm 1.32$ & $524.46 \pm 2.39$ & $1.65 \pm 0.01$ & $0.46 \pm 0.02$ \\
\hline & & $\mathrm{P} \leq$ & 0.002 & 0.04 & 0.0001 & 0.0001 & 0.0007 & 0.0007 & 0.0001 & 0.0001 \\
\hline
\end{tabular}

Table (3-b): Effect of feeding different levels of curcumin on lipid profile of normal rats (mg\%)

\begin{tabular}{|c|c|c|c|c|c|c|c|c|c|c|}
\hline & & & Cholesterol & HDL-C & LDL-C & VLDL-C & $\begin{array}{l}\text { Triacyl- } \\
\text { glycerol }\end{array}$ & $\begin{array}{c}\text { Phospho- } \\
\text { Lipids }\end{array}$ & $\frac{\text { Cholesterol }}{\text { HDL-C }}$ & $\frac{\text { LDL-C }}{\text { HDL-C }}$ \\
\hline 1 & Control I & $\mathrm{M} \pm \mathrm{SE}$ & $83.39 \pm 1.19$ & $44.84 \pm 0.87$ & $26.71 \pm 0.72$ & $11.82 \pm 0.36$ & $102.47 \pm 1.19$ & $541.72 \pm 3.48$ & $1.86 \pm 0.02$ & $0.60 \pm 0.02$ \\
\hline \multirow{2}{*}{7} & \multirow{2}{*}{ Curcumin } & $\mathrm{M} \pm \mathrm{SE}$ & $80.40 \pm 2.11$ & $42.33 \pm 0.94$ & $26.38 \pm 1.23$ & $11.69 \pm 0.36$ & $99.18 \pm 1.31$ & $534.78 \pm 5.93$ & $1.90 \pm 0.028$ & $0.62 \pm 0.025$ \\
\hline & & $\mathrm{P} \leq$ & NS & NS & NS & NS & NS & NS & NS & NS \\
\hline \multirow{2}{*}{8} & \multirow{2}{*}{ Curcumin } & $\mathrm{M} \pm \mathrm{SE}$ & $78.98 \pm 1.20$ & $41.80 \pm 0.92$ & $25.59 \pm 1.16$ & $11.54 \pm 0.45$ & $.98 .31 \pm 1.95$ & $532.04 \pm 5.31$ & $1.89 \pm 0.03$ & $0.61 \pm 0.028$ \\
\hline & & $\mathrm{P} \leq$ & NS & NS & NS & NS & NS & NS & NS & NS \\
\hline \multirow{2}{*}{9} & \multirow{2}{*}{ Curcumin } & $\mathrm{M} \pm \mathrm{SE}$ & $79.24 \pm 1.25$ & $47.66 \pm 0.76$ & $21.65 \pm 0.78$ & $9.93 \pm 0.26$ & $97.89 \pm 1.85$ & $531.86 \pm 5.56$ & $1.66 \pm 0.02$ & $0.45 \pm 0.01$ \\
\hline & & $\mathrm{P} \leq$ & 0.03 & 0.03 & 0.0001 & 0.0005 & NS & NS & 0.0001 & 0.0001 \\
\hline \multirow{2}{*}{10} & \multirow{2}{*}{ Curcumin } & $\mathrm{M} \pm \mathrm{SE}$ & $79.03 \pm 1.03$ & $47.58 \pm 0.56$ & $21.56 \pm 0.54$ & $9.89 \pm 0.27$ & $96.46 \pm 1.60$ & $526.84 \pm 3.44$ & $1.66 \pm 0.01$ & $0.45 \pm 0.01$ \\
\hline & & $\mathrm{P} \leq$ & 0.01 & 0.02 & 0.0001 & 0.0005 & 0.007 & 0.007 & 0.0001 & 0.0001 \\
\hline \multirow{2}{*}{11} & \multirow{2}{*}{ Curcumin } & $\mathrm{M} \pm \mathrm{SE}$ & $79.17 \pm 0.99$ & $47.65 \pm 0.58$ & $21.45 \pm 0.57$ & $9.87 \pm 0.21$ & $94.24 \pm 1.31$ & $522.65 \pm 2.29$ & $1.66 \pm 0.01$ & $0.45 \pm 0.01$ \\
\hline & & $\mathrm{P} \leq$ & 0.014 & 0.01 & 0.0001 & 0.0002 & 0.0002 & 0.0002 & 0.0001 & 0.0001 \\
\hline
\end{tabular}


Effect of Curcumin, Mixture of Curcumin and..........

Table (4-a): Effect of feeding different levels of curcumin on lipid profile of hypercholesterolemic rats $(\mathrm{mg} \%)$

\begin{tabular}{|c|c|c|c|c|c|c|c|c|c|c|}
\hline & & & Cholesterol & HDL-C & LDL-C & VLDL-C & $\begin{array}{l}\text { Triacyl- } \\
\text { glycerol }\end{array}$ & $\begin{array}{l}\text { Phospho } \\
\text { Lipids }\end{array}$ & $\frac{\text { Cholesterol }}{\text { HDL-C }}$ & $\frac{\mathrm{HDL}-\mathrm{C}}{\mathrm{LDL}-\mathrm{C}}$ \\
\hline $1^{1}$ & Control I & $\mathrm{M} \pm \mathrm{SE}$ & $83.39 \pm 1.19$ & $44.84 \pm 0.87$ & $26.71 \pm 0.72$ & $11.82 \pm 0.36$ & $102.47 \pm 1.19$ & $541.72 \pm 3.48$ & $1.86 \pm 0.015$ & $1.69 \pm 0.051$ \\
\hline \multirow{2}{*}{26} & \multirow{2}{*}{ Control II } & $\mathrm{M} \pm \mathrm{SE}$ & $174.93 \pm 3.08$ & $24.21 \pm 0.62$ & $105.61 \pm 2.1$ & $45.11 \pm 1.36$ & $201.62 \pm 7.02$ & $985.14 \pm 12.9$ & $7.24 \pm 0.103$ & $0.23 \pm 0.004$ \\
\hline & & $\left(26\right.$ vs $\left.1^{1}\right) P \leq$ & 0.0001 & 0.0001 & 0.0001 & 0.0001 & 0.0001 & 0.0001 & 0.0001 & 0.0001 \\
\hline \multirow{3}{*}{12} & \multirow{3}{*}{ Control III } & $\mathrm{M} \pm \mathrm{SE}$ & $144.09 \pm 2.95$ & $37.5 \pm 3.99$ & $75.32 \pm 1.57$ & $31.27 \pm 1.17$ & $154.43 \pm 5.44$ & $658.37 \pm 12.8$ & $3.87 \pm 0.099$ & $0.50 \pm 0.017$ \\
\hline & & $\left(12\right.$ vs $\left.1^{1}\right) \mathrm{P} \leq$ & 0.0001 & 0.0001 & 0.0001 & 0.0001 & 0.0001 & 0.0001 & 0.0001 & 0.0001 \\
\hline & & $(12$ vs 26$) \mathrm{P} \leq$ & 0.0001 & 0.0001 & 0.0001 & 0.0001 & 0.0001 & 0.0001 & 0.0001 & 0.0001 \\
\hline \multirow{4}{*}{13} & \multirow{4}{*}{ Curcumin } & $\mathrm{M} \pm \mathrm{SE}$ & $160.53 \pm 2.77$ & $26.94 \pm 1.33$ & $95.32 \pm 1.32$ & $38.27 \pm 1.59$ & $171.11 \pm 3.08$ & $820.57 \pm 8.45$ & $6.06 \pm 0.244$ & $0.28 \pm 0.017$ \\
\hline & & $\left(13\right.$ vs $\left.1^{1}\right) \mathrm{P} \leq$ & 0.0001 & 0.0001 & 0.0001 & 0.0001 & 0.0001 & 0.0001 & 0.0001 & 0.0001 \\
\hline & & $(13$ vs 26$) \mathrm{P} \leq$ & 0.003 & 0.049 & 0.0006 & 0.004 & 0.0009 & 0.0001 & 0.0003 & 0.006 \\
\hline & & $(13$ vs 12$) \mathrm{P} \leq$ & 0.0007 & 0.0001 & 0.0001 & 0.002 & 0.0001 & 0.0001 & 0.0001 & 0.0001 \\
\hline \multirow{4}{*}{14} & \multirow{4}{*}{ Curcumin } & $\mathrm{M} \pm \mathrm{SE}$ & $139.76 \pm 2.56$ & $33.27 \pm 1.31$ & $82.41 \pm 1.90$ & $24.11 \pm 0.93$ & $134.85 \pm 4.65$ & $684.72 \pm 13.6$ & $4.24 \pm 0.123$ & $0.41 \pm 0.019$ \\
\hline & & $\left(14\right.$ vs $\left.1^{1}\right) \mathrm{P} \leq$ & 0.0001 & 0.0001 & 0.0001 & 0.0001 & 0.0001 & 0.0001 & 0.0001 & 0.0001 \\
\hline & & $(14$ vs 26$) \mathrm{P} \leq$ & 0.0001 & 0.0001 & 0.0001 & 0.0001 & 0.0001 & 0.0001 & 0.0001 & 0.0001 \\
\hline & & $(14$ vs 12$) \mathrm{P} \leq$ & NS & 0.032 & 0.01 & 0.00015 & 0.013 & NS & 0.03 & 0.002 \\
\hline \multirow{4}{*}{15} & \multirow{4}{*}{ Curcumin } & $\mathrm{M} \pm \mathrm{SE}$ & $108.12 \pm 2.18$ & $41.41 \pm 1.30$ & $48.15 \pm 0.82$ & $18.56 \pm 0.70$ & $120.98 \pm 3.04$ & $603.17 \pm 9.14$ & $2.62 \pm 0.04$ & $0.86 \pm 0.03$ \\
\hline & & $\left(15\right.$ vs $\left.1^{1}\right) P \leq$ & 0.0001 & 0.042 & 0.0001 & 0.0001 & 0.0001 & 0.0001 & 0.0001 & 0.0001 \\
\hline & & $(15$ vs 26$) \mathrm{P} \leq$ & 0.0001 & 0.0001 & 0.0001 & 0.0001 & 0.0001 & 0.0001 & 0.0001 & 0.0001 \\
\hline & & $(15$ vs 12$) P \leq$ & 0.0001 & 0.045 & 0.0001 & 0.0001 & 0.0001 & 0.0025 & 0.0001 & 0.0001 \\
\hline \multirow{4}{*}{16} & \multirow{4}{*}{ Curcumin } & $\mathrm{M} \pm \mathrm{SE}$ & $100.39 \pm 2.33$ & $42.01 \pm 0.96$ & $42.31 \pm 1.38$ & $16.07 \pm 0.66$ & $112.18 \pm 4.67$ & $586.17 \pm 10.2$ & $2.39 \pm 0.02$ & $1.00 \pm 0.02$ \\
\hline & & $\left(16\right.$ vs $\left.1^{1}\right) \mathrm{P} \leq$ & 0.0001 & 0.04 & 0.0001 & 0.0001 & NS & 0.0006 & 0.0001 & 0.0001 \\
\hline & & $(16$ vs 26$) \mathrm{P} \leq$ & 0.0001 & $7.17 \mathrm{E}-12$ & 0.0001 & 0.0001 & 0.0001 & 0.0001 & 0.0001 & 0.0001 \\
\hline & & $(16$ vs 12$) P \leq$ & 0.0001 & 0.011 & 0.0001 & 0.0001 & 0.0001 & 0.0003 & 0.0001 & 0.0001 \\
\hline \multirow{4}{*}{17} & \multirow{4}{*}{ Curcumin } & $\mathrm{M} \pm \mathrm{SE}$ & $90.06 \pm 3.26$ & $42.86 \pm 2.07$ & $33.55 \pm 0.90$ & $13.65 \pm 0.61$ & $109.21 \pm 1.96$ & $556.13 \pm 8.43$ & $2.12 \pm 0.046$ & $1.28 \pm 0.05$ \\
\hline & & $\left(17\right.$ vs $\left.1^{1}\right) \mathrm{P} \leq$ & NS & NS & 0.0001 & 0.02 & 0.009 & NS & 0.0001 & 0.0001 \\
\hline & & $(17$ vs 26$) \mathrm{P} \leq$ & 0.0001 & 0.0001 & 0.0001 & 0.0001 & 0.0001 & 0.0001 & 0.0001 & 0.0001 \\
\hline & & (17 vs 12$) \mathrm{P} \leq$ & 0.0001 & 0.04 & 0.0001 & 0.0001 & 0.0001 & 0.0001 & 0.0001 & 0.0001 \\
\hline
\end{tabular}

Table (4-b): Effect of feeding different levels of Curcuminin+piperine on lipid profile of hypercholesterolemic rats $(\mathrm{mg} \%)$

\begin{tabular}{|c|c|c|c|c|c|c|c|c|c|c|}
\hline & & & Cholesterol & HDL-C & LDL-C & VLDL-C & $\begin{array}{l}\text { Triacyl- } \\
\text { glycerol }\end{array}$ & Phospho-Lipids & $\frac{\text { Cholesterol }}{\text { HDL-C }}$ & $\frac{\text { LDL-C }}{\mathrm{HDL}-\mathrm{C}}$ \\
\hline $1^{1}$ & Control I & $\mathrm{M} \pm \mathrm{SE}$ & $83.39 \pm 1.19$ & $44.84 \pm 0.87$ & $26.71 \pm 0.72$ & $11.82 \pm 0.36$ & $102.47 \pm 1.19$ & $541.72 \pm 3.48$ & $1.86 \pm 0.015$ & $0.60 \pm 0.02$ \\
\hline 26 & Control I & $\mathrm{M} \pm \mathrm{SE}$ & $174.93 \pm 3.08$ & $24.21 \pm 0.62$ & $105.61 \pm 2.1$ & $45.11 \pm 1.36$ & $201.62 \pm 7.02$ & $985.14 \pm 12.9$ & $7.24 \pm 0.103$ & $4.37 \pm 0.071$ \\
\hline 12 & Control II & $\mathrm{M} \pm \mathrm{SE}$ & $144.09 \pm 2.95$ & $37.5 \pm 3.99$ & $75.32 \pm 1.57$ & $31.27 \pm 1.17$ & $154.43 \pm 5.44$ & $658.37 \pm 12.8$ & $3.87 \pm 0.099$ & $2.02 \pm 0.063$ \\
\hline \multirow{4}{*}{18} & \multirow{4}{*}{$\begin{array}{c}\text { Curcumin } \\
+ \\
\text { Piperine }\end{array}$} & $\mathrm{M} \pm \mathrm{SE}$ & $149.67 \pm 2.48$ & $30.58 \pm 1.39$ & $84.77 \pm 1.11$ & $34.32 \pm 1.85$ & $160.99 \pm 3.07$ & $716.06 \pm 9.53$ & $4.96 \pm 0.18$ & $2.83 \pm 0.14$ \\
\hline & & $\left(18\right.$ vs $\left.1^{1}\right) P \leq$ & 0.0001 & 0.0001 & 0.0001 & 0.0001 & 0.0001 & 0.0001 & 0.0001 & 0.0001 \\
\hline & & $(18$ vs 26$) \mathrm{P} \leq$ & 0.0001 & 0.0006 & 0.0001 & 0.0002 & 0.0001 & 0.0001 & 0.0001 & 0.0001 \\
\hline & & $(18$ vs 12$) \mathrm{P} \leq$ & NS & 0.002 & 0.0001 & NS & NS & 0.002 & 0.0001 & 0.0001 \\
\hline \multirow{4}{*}{19} & \multirow{4}{*}{$\begin{array}{c}\text { Curcumin } \\
+ \\
\text { Piperine }\end{array}$} & $\mathrm{M} \pm \mathrm{SE}$ & $122.06 \pm 2.19$ & $36.68 \pm 1.26$ & $70.22 \pm 1.21$ & $15.16 \pm 0.46$ & $120.85 \pm 3.71$ & $627.12 \pm 12.0$ & $3.35 \pm 0.07$ & $1.93 \pm 0.07$ \\
\hline & & $\left(19\right.$ vs $\left.1^{1}\right) P \leq$ & 0.0001 & 0.0001 & 0.0001 & 0.0001 & 0.0002 & 0.0001 & 0.0001 & 0.0001 \\
\hline & & $(19$ vs 26$) \mathrm{P} \leq$ & 0.0001 & 0.0001 & 0.0001 & 0.0001 & 0.0001 & 0.0001 & 0.0001 & 0.0001 \\
\hline & & $(19$ vs 12$) \mathrm{P} \leq$ & 0.0001 & NS & 0.02 & 0.0001 & 0.0001 & NS & 0.0006 & NS \\
\hline \multirow{4}{*}{20} & \multirow{4}{*}{$\begin{array}{c}\text { Curcumin } \\
+ \\
\text { Piperine }\end{array}$} & $\mathrm{M} \pm \mathrm{SE}$ & $100.42 \pm 1.87$ & $42.92 \pm 0.90$ & $45.15 \pm 1.14$ & $12.35 \pm 0.56$ & $103.08 \pm 2.58$ & $558.77 \pm 8.33$ & $2.34 \pm 0.03$ & $1.05 \pm 0.02$ \\
\hline & & $\left(20\right.$ vs $\left.1^{1}\right) P \leq$ & 0.0001 & NS & 0.0001 & NS & NS & NS & 0.0001 & 0.0001 \\
\hline & & $(20$ vs 26$) \mathrm{P} \leq$ & 0.0001 & 0.0001 & 0.0001 & 0.0001 & 0.0001 & 0.0001 & 0.0001 & 0.0001 \\
\hline & & $(20$ vs 12$) P \leq$ & 0.0001 & 0.0026 & 0.0001 & 0.0001 & 0.0001 & 0.0001 & 0.0001 & 0.0001 \\
\hline
\end{tabular}


Table (4-c): Effect of feeding different levels of curcum on lipid profile of hypercholesterolemic rats $(\mathbf{m g} \%)$

\begin{tabular}{|c|c|c|c|c|c|c|c|c|c|c|}
\hline & & & Cholesterol & HDL-C & LDL-C & VLDL-C & $\begin{array}{l}\text { Triacyl- } \\
\text { glycerol }\end{array}$ & Phospho-Lipids & $\frac{\text { Cholesterol }}{\text { HDL-C }}$ & $\frac{\mathrm{LDL}-\mathrm{C}}{\mathrm{HDL}-\mathrm{C}}$ \\
\hline $1^{1}$ & Control I & $\mathrm{M} \pm \mathrm{SE}$ & $83.39 \pm 1.19$ & $44.84 \pm 0.87$ & $26.71 \pm 0.72$ & $11.82 \pm 0.36$ & $102.47 \pm 1.19$ & $541.72 \pm 3.48$ & $1.86 \pm 0.015$ & $0.60 \pm 0.02$ \\
\hline 26 & Control II & $\mathrm{M} \pm \mathrm{SE}$ & $174.93 \pm 3.08$ & $24.21 \pm 0.62$ & $105.61 \pm 2.1$ & $45.11 \pm 1.36$ & $201.62 \pm 7.02$ & $985.14 \pm 12.9$ & $7.24 \pm 0.103$ & $4.37 \pm 0.071$ \\
\hline 12 & Control III & $\mathrm{M} \pm \mathrm{SE}$ & $144.09 \pm 2.95$ & $37.5 \pm 3.99$ & $75.32 \pm 1.57$ & $31.27 \pm 1.17$ & $154.43 \pm 5.44$ & $658.37 \pm 12.8$ & $3.87 \pm 0.099$ & $2.02 \pm 0.063$ \\
\hline \multirow{4}{*}{2} & \multirow{4}{*}{ Curcum } & $\mathrm{M} \pm \mathrm{SE}$ & $156.32 \pm 2.65$ & $25.98 \pm 1.24$ & $93.08 \pm 1.58$ & $37.26 \pm 1.70$ & $168.78 \pm 2.75$ & $817.39 \pm 7.79$ & $6.12 \pm 0.254$ & $3.67 \pm 0.197$ \\
\hline & & $\left(21\right.$ vs $\left.1^{1}\right) \mathrm{P} \leq$ & 0.0001 & 0.0001 & 0.0001 & 0.0001 & 0.0001 & 0.0001 & 0.0001 & 0.0001 \\
\hline & & (21 vs 26$) \mathrm{P} \leq$ & 0.0002 & NS & 0.00015 & 0.002 & 0.0004 & 1.62E-09 & 0.0007 & 0.003 \\
\hline & & (21 vs 12$) \mathrm{P} \leq$ & 0.006 & 0.0001 & 0.0001 & 0.0095 & 0.03 & 0.0001 & 0.0001 & 0.0001 \\
\hline \multirow{4}{*}{22} & \multirow{4}{*}{ Curcum } & $\mathrm{M} \pm \mathrm{SE}$ & $137.48 \pm 2.05$ & $32.27 \pm 1.06$ & $81.63 \pm 1.86$ & $23.58 \pm 0.92$ & $130.82 \pm 4.33$ & $679.97 \pm 14.0$ & $4.29 \pm 0.119$ & $2.56 \pm 0.113$ \\
\hline & & $\left(22\right.$ vs $\left.1^{1}\right) \mathrm{P} \leq$ & 0.0001 & 0.0001 & 0.0001 & 0.0001 & 0.0001 & 0.0001 & 0.0001 & 0.0001 \\
\hline & & (22 vs 26$) \mathrm{P} \leq$ & 0.0001 & 0.0001 & 0.0001 & 0.0001 & 0.0001 & 0.0001 & 0.0001 & 0.0001 \\
\hline & & (22 vs 12$) \mathrm{P} \leq$ & NS & 0.0052 & 0.02 & 0.0001 & 0.002 & NS & 0.01 & 0.0006 \\
\hline \multirow{4}{*}{23} & \multirow{4}{*}{ Curcum } & $\mathrm{M} \pm \mathrm{SE}$ & $114.83 \pm 1.99$ & $36.95 \pm 1.24$ & $60.56 \pm 0.85$ & $17.32 \pm 0.52$ & $117.68 \pm 2.86$ & $600.54 \pm 9.35$ & $3.13 \pm 0.067$ & $1.66 \pm 0.059$ \\
\hline & & $\left(23\right.$ vs $\left.1^{1}\right) P \leq$ & 0.0001 & 0.0001 & 0.0001 & 0.0001 & 0.0001 & 0.0001 & 0.0001 & 0.0001 \\
\hline & & (23 vs 26$) \mathrm{P} \leq$ & 0.0001 & 0.0001 & 0.0001 & 0.0001 & 0.0001 & 0.0001 & 0.0001 & 0.0001 \\
\hline & & (23 vs 12$) \mathrm{P} \leq$ & 0.0001 & NS & 0.0001 & 0.0001 & 0.0001 & 0.0018 & 0.0001 & 0.0005 \\
\hline \multirow{4}{*}{24} & \multirow{4}{*}{ Curcum } & $\mathrm{M} \pm \mathrm{SE}$ & $97.92 \pm 1.89$ & $39.47 \pm 0.86$ & $42.92 \pm 1.09$ & $15.53 \pm 0.58$ & $110.76 \pm 4.36$ & $583.46 \pm 10.3$ & $2.48 \pm 0.025$ & $1.09 \pm 0.024$ \\
\hline & & $\left(24\right.$ vs $\left.1^{1}\right) P \leq$ & 0.0001 & 0.0003 & 0.0001 & 0.0001 & NS & 0.001 & 0.0001 & 0.0001 \\
\hline & & (24 vs 26$) \mathrm{P} \leq$ & 0.0001 & 0.0001 & 0.0001 & 0.0001 & 0.0001 & 0.0001 & 0.0001 & 0.0001 \\
\hline & & (24 vs 12$) \mathrm{P} \leq$ & 0.0001 & NS & 0.0001 & 0.0001 & 0.0001 & 0.0002 & 0.0001 & 0.0001 \\
\hline \multirow{4}{*}{25} & \multirow{4}{*}{ Curcum } & $\mathrm{M} \pm \mathrm{SE}$ & $88.6 \pm 3.13$ & $42.26 \pm 2.25$ & $33.12 \pm 0.53$ & $13.22 \pm 0.60$ & $106.37 \pm 1.59$ & $553.54 \pm 8.11$ & $2.12 \pm 0.047$ & $0.80 \pm 0.04$ \\
\hline & & $\left(25\right.$ vs $\left.1^{1}\right) \mathrm{P} \leq$ & NS & NS & 0.0001 & NS & NS & NS & 0.0001 & 0.0002 \\
\hline & & (25 vs 26$) \mathrm{P} \leq$ & 0.0001 & 0.0001 & 0.0001 & 0.0001 & 0.0001 & 0.0001 & 0.0001 & 0.0001 \\
\hline & & (25 vs 12$) \mathrm{P} \leq$ & 0.0001 & NS & 0.0001 & 0.0001 & 0.0001 & 0.0001 & 0.0001 & 0.0001 \\
\hline
\end{tabular}

Table (5-a): Lipid profile of normal rats' liver and heart fed different levels of curcum or curcumin $(\mathbf{m g} / \mathbf{1 0 0 g})$

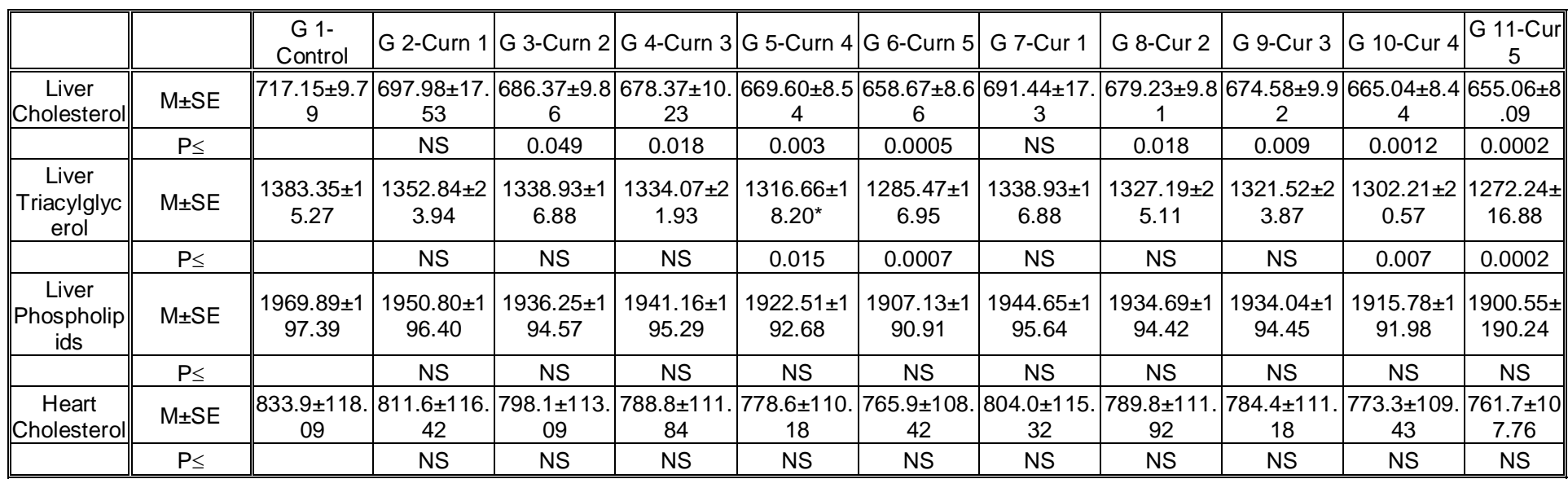


Table (5-b): Lipid profile of rats' liver and heart of hypercholesterolemic rats fed different levels of curcum or curcumin or curcumin+piperine $(\mathrm{mg} / 100 \mathrm{~g})$

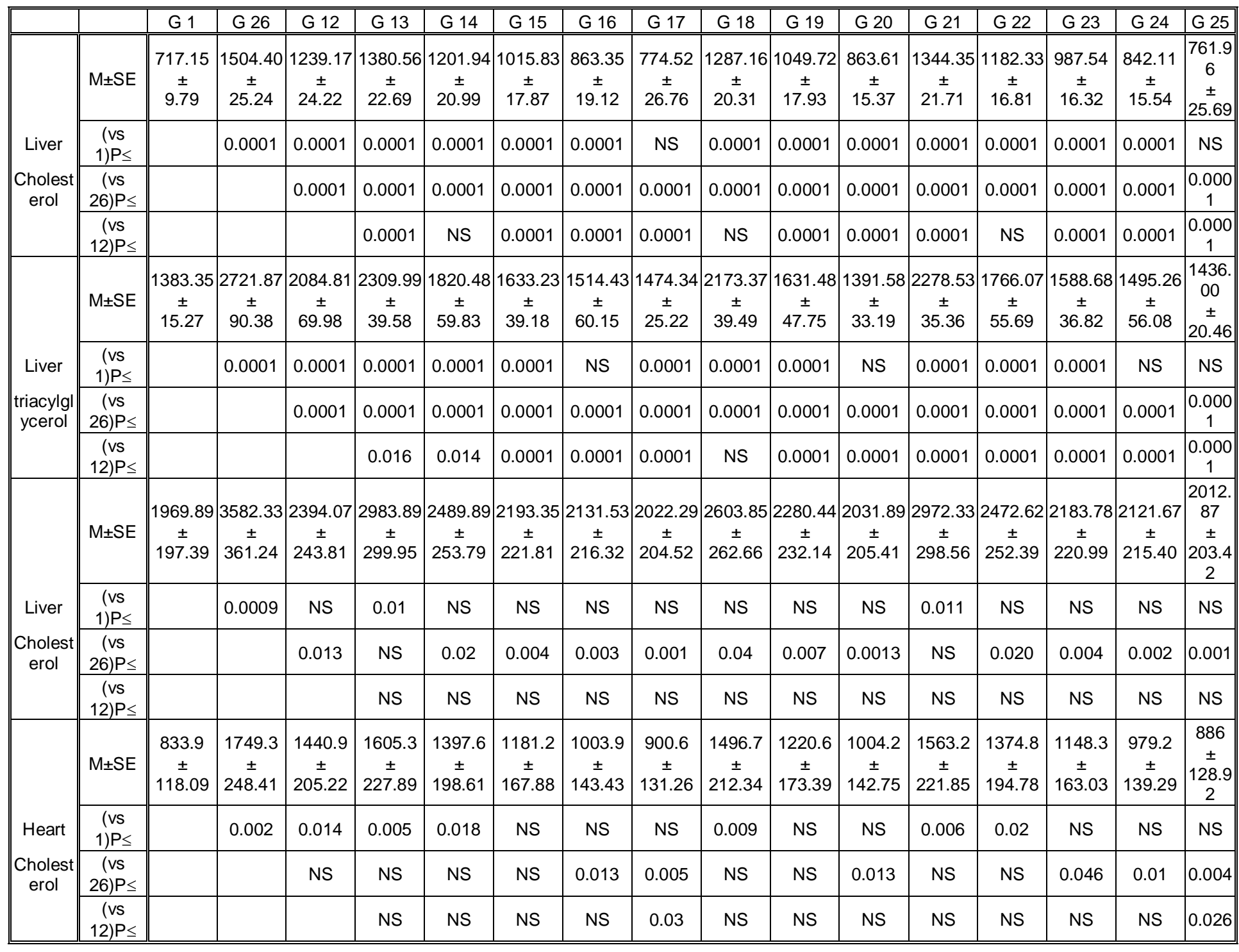

\section{Discussion}

BWG of cholesterol fed rats was significantly higher than that of their respective control group or their respective initial groups, which are in agreement with Hulbron et al. (1982) and disagree with Sérougne et al. (1995).

The significant increase of liver and heart weight may be attributed to fat deposit (Table 2-a). As recorded by several investigators, increased cholesterol level increases the fat accumulation in liver (Kahlone et al. 1997 and Murray et al. 2000).
Table (3-a, b) revealed that serum cholesterol (total, LDL, VLDL) of groups (G: 4-6 and 9-11) of curcumin- or curcumfed groups was significantly lower than control group. Also serum HDL-C of groups (G: 4-6 and 9-11) of curcumin- or curcum- fed groups was significantly higher than control group. Cholesterol decrease occurs in LDL and VLDL fraction $(\approx 20 \%$ each). Soni and Kuttan (1992) found that oral curcumin given to healthy human volunteers resulted in a significant decrease in the level of serum lipid 
peroxides $(33 \%)$, significant increase in HDL-C (29\%), and a significant decrease in total serum cholesterol (11.63\%).

Tables $(4-a, b, c)$ revealed that serum cholesterol (total, LDL, VLDL); triacylglycerol and phospholipids levels of cholesterol-fed groups were significantly higher than control group, while HDL-C was significantly lower than control group. The significant increase in serum cholesterol level may be due to type and amount of oil in which cholesterol was dissolved (Grundy and Denke 1990 and Tebib et al. 1994). Serum total cholesterol was significantly higher in cholesterol-fed rats due substantially to greater VLDL concentration due to increased intery of cholesterol into the circulation (Abbey et al. 1993) and suppression of cholesterol transport by HDL. The significant increase in serum LDL-C levels may be due to suppression of LDL receptor activity in cholesterol-fed rats (Sorci-Thomas et al. 1989), which allow for an increased conversion of VLDL remnants to LDL since VLDL remnants are partially removed by LDL receptors. This will lead to an over production of LDL (Kovanen et al. 1981 and Sorci-Thomas et al. 1989). The data also showed that using $0.5 \%$ level ( $\mathrm{G} \mathrm{15,23)}$ is better than using $2.0 \%$ level which is $4 x(G 23,25)$ since degree of change does not go with using this high level. Curcum is slightly better than curcumin as hypocholes-terolemic agent. Curcumin reduces choles-terol by interfering with intestinal choles-terol uptake, increasing the conversion of cholesterol into bile acids, and increasing the excretion of bile acids or through interfering with exogenous cholesterol absorption (Srinivasan and Sambaiah 1991). Also HDL cleans off the walls of blood vessels, thus removing excess cholesterol, LDL. The HDL then carries this cholesterol to the liver where it is processed, or by decreasing thiobarbityric acid (TBA) value and improved total antioxidation capability. Also through enhancing the activities of SOD (superoxide dismutase) and GSH-PX in liver (Wang et al. 2000). The hypocholesterolemic effect may also be due to the significantly increased activity of hepatic
acyl-CoA oxidase of treated hypercholesterolemic rats. Furthermore, epididymal adipose tissue weight was significantly reduced with curcuminoid intake in a dosedependent manner, which means that dietary curcuminoids have lipid-lowering potency in vivo, probably due to alterations in fatty acid metabolism (Asai and Miyazawa 2001). Babu and Srinivasan (1997) suggested that the hypocholesterolemic activity is properly due to increased hepatic cholesterol-7ahydroxylase activity thus leading to higher rate of cholesterol catabolism. Soudamini et al. (1992) and Sreejayan and Rao (1994) stated that curcumin and curcumoniods act as inhibitors of lipid peroxidation which lead to cholesterol reduction. The results agree with Patil and Srinivasan (1971); Soudamini et al. (1992), and Sreejayan and Rao (1994); And disagree with Deters et al. (2001) who conclude that curcumin is not able to prevent cyclosporine-induced cholestasis and hyperlipidemia after prolonged administration in bile fistula rats. Curcumin or curcum lower cholesterol, LDL-C, VLDL-C and raising HDL-C, which act as independent factor. The National cholesterol Education Program's new guidelines now recognize that low HDL levels as a strong independent risk factor for coronary artery disease and recommend raising HDL, which is another important factor to reduce risk of heart disease.

Pharmacokinetic properties of curcumin indicate that curcumin when given orally is less active, this may be because it is absorbed poorly by the gastrointestinal tract and/or underlies presystemic transformation since after oral application only traces of curcumin were found in the blood and this may be due to transformation of curcumin into unidentified compounds in absorption and its glucuronidation in the liver, and that, on the other hand, most of the curcumin is excreted via the faeces (Ravindranath and Chandrasekhara 1980 and 1981 and Ammon and Wahl 1991). Due to the low bioavailabilty of curcumin, piperine (1 piperoylpiperidine), active ingredients of black pepper (Piper nigrum L.) and others, were added to curcumin to enhance its bioavailabilty by 
increasing absorption and reducing its metabolism in liver (Shoba et al. 1997) since piperine is known to enhance drugs by inhibition of glucuronidation in the liver and small intestine (Atal et al. 1985 and Singh et al. 1986).

Regarding atherogenic indices, it agrees with Dixit et al. (1988), who found that extract of Curcuma longa $L$. reduces significantly HDL-C/cholesterol ratio.

Significantly higher levels of hepatic cholesterol, triacylglycerol and phospholipids concentrations; and cardiac cholesterol concentration in cholesterol-fed group (G 26) or the untreated hypercholesterolemic control group ( $G$ 12) than control group was found. The reason for the higher hepatic cholesterol, triacylglycerol and phospholipids concentrations as reported by Fungwe et al. (1993) may be as a result of addition cholesterol to rats' diet, which leads to stimulation of the synthesis of triacylglycerol and/or reduced fatty acid synthesis. Dietary cholesterol may stimulate biosynthesis of triacylglycerol through increased activity of glycerophosphate acylt-ransferase which catalyze the first comm-itted step in glycerolipid synthesis, phosp-hatide phosphohydrolase which control the rate of diglyceride production and diacyltransferase which may regulate the channeling of diglyceride into triglyceride (Fukada and Ontko 1984, Fungwe et al. 1993 and Liu et al. 1995). The significantly higher cardiac cholesterol concentration agrees with Hulbron et al. (1982) and KrisEtherton et al. (1984) and it reflects sensibility of heart to cholesterol feeding.

Significantly lower levels of hepatic cholesterol, triacylglycerol concentrations; and cardiac cholesterol concentration in almost all groups (G: 13-17, 18-20, 21-25) groups when compared with $\left(\mathrm{G} 1^{1}, 12,26\right)$ was found. These results agree with Asai and Miyazawa (2001). The change may be because dietary turmeric lowers lipid peroxi-dation by enhancing the activities of antioxidant enzymes (Reddy and Lokesh 1994). The decrease in hepatic triacylglycerol as proposed by Asai and Miyazawa (2001) is that curcuminor curcuminoids affect fatty acid catabolism rather than de novo synthesis through multiple induction of intra- and extra cellular fatty acid catabolism and utilization pathways e. g. induction of fatty acid $\beta$-oxidation and triacylglycerol hydrolysis with metabolites of absorbed curcuminoids serving as ligands that can activate PPARs (peroxisome proliferator-activated receptors) which regulate gene expression of ACO (acetyl CoA oxidase) which perform the first catalytic steps enzyme of peroxisomal fatty acid $\beta$-oxidation (Pan et al. 1999; Ramirez-Tortosa et al. 1999; Asai and Miyazawa 2000; Ireson et al.2001).

\section{Conclusion}

The use of curcum, curcumin, and mixture of curcumin and piperine may be useful in the management of cardiovascular disease. Using the lower level $(0.5 \%)$ is better than using $2.0 \%$. Piperine enhances the bioavailabilty of curcumin.

\section{References}

1. Abbey M, Triantafilidis C, and Topping DL. (1993): Dietary non-starch polysaccharides interact with cholesterol and fish oil in their effect on plasma lipids and hepatic lipoprotein receptor activity in rats. J. Nut., 123: 900-908.

2. Aggarwal BB, Kumar A, and Bharti AC. (2003): Anticancer potential of curcumin: preclinical and clinical studies. Anticancer Res. 2003; 23(1A): 363-398.

3. Allain CC, Poor LS, Chan CSG, Richmond W, and Fu PC. (1974): Enzymatic determination of total serum cholesterol. Clin. Chem., 20: 470-475.

4. Ammon HP, and Whal MA. (1991): Pharmacology of Curcuma longa. Planta Med., 57: 1-7.

5. AOAC (1981): Official Methods of Analysis of the Association of Official Analytical Chemists. Published by the Association of Analytical chemists Inc. $10^{\text {th }}$ Ed., Edited by Kenneth helrich. Page 56. Arlington, U. S. A.

6. Asai A, and Miyazawa T. (2000): Occurrence of orally administered curcuminoid as glucuronide and glucuronide/ sulfate conjugates in rat plasma. Life Sci.; 67(23): 2785-2793.

7. Asai A, and Miyazawa T. (2001): Dietary curcuminoids prevent high-fat diet-induced lipid accumulation in rat liver and 
epididymal adipose tissue. J Nutri.; 131(11):2932-2935.

8. Atal CK, Dubey RK, and Singh J. (1985): Biochemical basis of enhanced drug bioavailability by piperine: evidence that piperine is a potent inhibitor of drug metabolism. J. Pharmacol. Exp.Therap.; 232: 258-262.

9. Avram G. (1964): Quantitative Data. In: Biostatistics: An Introductory text. Chapter 2, page 53 \& 63. The MacMillan Company, New York, Collier MacMillan limited, London.

10. Babu PS, and Srinivasan P. (1997): Hypolipidemic action of curcumin, the active principle of turmeric Curcuma longa in streptozotocin induced diabetic rats. Mol. Cell Biochem., 166: 169-175.

11. Bharti AC, Donato N, Singh S, and Aggarwal BB. (2003): Curcumin (diferuloylmethane) down regulates the constitutive activation of nuclear factor kappa B and IkappaBalpha kinase in human multiple myeloma cells, leading to suppression of proliferation and induction of apoptosis. Blood, 101(3): 1053-1062.

12. Bratman S, and Girman AM. (2003): "Mosby's handbook of herbs and supplements and their therapeutic uses. Page 502503, Mosby Health Gate, St. Louis.

13. Bucolo G, and David H (1973): Quantitative determination of triglycerides by the use of enzymes. Clin chem., 19: 476-482.

14. Burstein M, Scholnick HR. and Monfin R. (1970): Rapid method for the isolation of lipoproteins from human serum by precipitation with polyanions. J. Lipid Res., 11: 585-595.

15. Carr T, Andresson CJ, and Rudel LL. (1993): Enzymatic determination of triglycerides, free cholesterol and total cholesterol in tissue lipid extracts. Clin. Chem., 26: 39-42.

16. Chan WH, Wu CC, and Yu JS. (2003): Curcumin inhibits UV-induced oxidative stress and apoptotic biochemical changes in human epidermoid carcinoma A431 cells. J. Cell Biochem.; 90: 327-338.

17. Cheng AL, Hsu CH, Lin JK, Hsu MM, Ho YF, Shen TS, Ko JY, Lin JT, Lin BR, Ming-Shiang W, Yu HS, Jee SH, Chen GS, Chen TM, Chen CA, Lai MK, Pu YS, Pan MH, Wang YJ, Tsai CC, and Hsieh CY. (2001): Phase I clinical trial of curcumin, a chemopreventive agent, in patients with high-risk or pre-malignant lesions. Anticancer Res.; 21(4B): 28952900.
18. Connerty HV, Briggs AR, and Eaton EH. JR. (1961): Simplified Determination of lipid component of blood serum. Clin. Chem., 7: 43-45.

19. Deters M, Klabunde T, Meyer H, Resch K, Kaever V. (2003): Effects of curcumin on cyclosporine-induced cholestasis and hypercholesterolemia and on cyclosporine metabolism in the rat. Planta Med.; 69(4): 337-343.

20. Dixit, VP, Jain P, and Joshi SC. (1988): Hypolipidaemic effects of Curcuma longa $L$ and Nardostachys jatamansi, DC in triton-induced hyperlipidaemic rats. Indian J Physiol. Pharmacol.; 32(4):299-304.

21. Folch J, Lees M, and Sloane Stanely GH. (1957): A simple method for the isolation and purification of total lipids from animal tissues. J. Biol. Chem., 226: 497-509.

22. Friedewald WT, Levy RI, and Fredrickson DS. (1972): Estimation of the concentration of low-Density lipoprotein cholesterol in plasma without use of the preparative ultra centrifuge. Clin. Chem., 18: 499-502.

23. Fruchart GC. (1982): LDL-Cholesterol determination after separation of low density lipoprotein. Rev. Fr. Des. Lab.; 7: 103-117.

24. Fukoda N, and Ontko JA. (1984): Interactions between fatty acid synthesis, oxidation and esterifiction in the production of triglyceride rich lipoprotein by the liver. J. Lipid Res., 25: 831-842.

25. Fungwe TV, Cagen LM, Cook GA, Wilcox HG, and Heimberg M. (1993): Dietary cholesterol stimulates hepatic biosynthesis of triglycerides and reduces oxidation of fatty acids in the rat. J. Lipid. Res., 34: 933-941.

26. Grundy SM, and Denke MA. (1990): Dietary Influences on serum lipids and lipoproteins. J. Lipid Res.; 31: 1149-1172.

27. Hulbron G, Aubert R, Bourgeois F, and Lemonnier D. (1982): Early cholesterol feeding: Are there long-term effects in the rats?. J. Nut., 112: 1246-1305.

28. Ireson C, Orr S, Jones DJ, Verschoyle R, Lim CK, Luo, JL, Howells L, Plummer S, Jukes R, Williams M, Steward WP, and Gescher A. (2001): Characterization of metabolites of the chemopreventive agent curcumin in human and rat hepatocytes and in the rat in vivo, and evaluation of their ability to inhibit phorbol ester-induced prostaglandin E2 production. Cancer Res.; 61(3): 1058-1064.

29. Ivan AR. (1999): Medicinal plants of the world, Chemical constituents, Traditional 
and modern medicinal uses. Page 139-152, human press, N. J.

30. Kahlone TS, Chow FI, Irving DW, and Sayre RN. (1997): Cholesterol response and fatty streak formation in hamsters fed two levels of saturated fat and various levels of cholesterol. J. Nut. Res., 17: 16931707.

31. Kovanon PT, Brown MS, Basu SK, Bilheimer DW, and Goldstein JL. (1981): Saturation and suppression of hepatic lipoprotein receptors: A mechanism for the hypercholesterolemia of cholesterol-fed rabbits. Proc. Nat. Acad. Sci. USA., 78: 1396-1400.

32. Kris-Etherton PM, HO CY, and Fosmire MA. (1984): The effect of dietary fat saturation on plasma and hepatic lipoproteins in the rats. J. Nut., 114: 16751682.

33. Levy, R. I. (1981): Cholesterol lipoprotein, apolipoproteins, and heart disease: Present status and future properties. Clin. Chem., 27: 653-662.

34. Liu CH, Huang M, and Huang PC. (1995): Sources of triacylglycerol accumulation in livers of rats fed a cholesterol-supplemented diet. Lipids, 30: 527-531.

35. Lopes-Virella MF, stone PG, Ellis S, and Coldwell JA. (1977): Cholesterol determination in high density lipoprotein separated by three diffrenet method. Clin. Chem., 23: 882-884.

36. Mesa MD, Aguilera CM, RamirezTortosa CL, Ramirez-Tortosa MC, Quiles JL, Baró L, Martínez de Victoria E, and Gil A. (2003): Oral administration of a turmeric extract inhibits erythrocyte and liver microsome membrane oxidation in rabbits fed with an atherogenic diet. Nutrition; 19(9): 800-804.

37. Murray RR, Granner DK, Mayes PA, and Rodwell VW. (2000): Harpers' Biochemistry, chapter $52 \& 60$.

38. National Research Council (NRC) Committee on Animal Nutrition, (1978): Nutrient Requirement of Laboratory animals. No. 10. $3^{\text {rd }}$ Revised Edition. National academy of science, National Research Council, Washington, D. C.

39. Pan MH, Huang TM, and Lin JK. (1999): Biotransformation of curcumin through reduction and glucuronidation in mice. Drug Metab. Dispos.;27(4): 486-494.

40. Patil TN, and Srinivasan M. (1971): Hypocholesterolaemic effect of curcumin in induced hypocholesterolaemic rats. Indian J. Exp. Biol.; 9: 167-169.
41. Phan TT, See P, Lee ST, and Chan SY. (2001): Protective effects of curcumin against oxidative damage on skin cells in vitro: its implication for wound healing. J. Trauma; 51(5): 927-931.

42. Quiles JL, Mesa MD, Ramirez-Tortosa CL, Aguilera CM, Battino M, Gil A, and Ramirez-Tortosa MC. (2002): Curcuma longa extract supplementation reduces oxidative stress and attenuates aortic fatty acid streak development in rabbits. Arterioscler. Thromb Vasc. Biol.; 22(7): 1225-1231.

43. Ramirez-Boscá A, Soler A, Gutierrez MAC, Álvarez JL, and Almagro EQ. (1995): Antioxidant curcuma extracts decrease the blood lipid peroxide levels of human subjects. Age; 18: 167-169.

44. Ramirez-Tortosa MC, Mesa MD, Aguilera MC, Quiles JL, Baró L, Martínez de Victoria E, and Gil A. (1999): Oral administration of a turmeric extract inhibits LDL oxidation and has hypocholesterolemic effects in rabbits with experimental atherosclerosis. Atherosclerosis; 147(2): 371-378.

45. Ramsewak RS, DeWitt DL, and Nair MG. (2000): Cytotoxicity, antioxidant and anti-inflammatory activities of curcumins IIII from Curcuma longa. Phytomedicine; 7(4): 303-308.

46. Ravindranath $\mathbf{V}$, and Chandrasekhara N. (1980): Absorption and tissue distribution of curcumin in rats. Toxicology; 16: 259-260.

47. Ravindranath $\mathbf{V}$, and Chandrasekhara N. (1981): In vitro studies on the intestinal absorption of curcumin in rats. Toxicology; 20: 251-257.

48. Reddy AC, and Lokesh BR. (1994): Effect of dietary turmeric (Curcuma longa) on iron-induced lipid peroxidation in the rat liver. Food Chem. Toxicol.; 32(3): 279283.

49. Reeves PG, Nielson FH, and Fahey GC, Jr. (1993): Ain 93 Purified diets for laboratory rodents: Final report of the American Institute of Nutrition and HOC Wriling Committee on the Reformation of the Ain 76 A Rodent Diet. J. Nut., 123: 1939-1952.

50. Richmond W. (1973): Preparation and properties of cholesterol oxidase from Nocardia Sp. And its application to the enzymatic assay of total cholesterol in serum. Clin. Chem., 19: 1350-1356.

51. Saleheen D, Ali SA, Ashfaq K, Siddiqui AA, Agha A, and Yasinzai MM. (2002): Latent activity of curcumin against 
leishmaniasis in vitro. Biol. Pharm. Bull.; 25(3): 386-389.

52. Sérougne C, Catherine F, Jacqueleni F, Tahar H, Catherine B, and Andrzej M. (1995): Hypercholesterolemia induced by cholesterol or cysteine-enriched diets are characterized by different concentrations in rats. J. Nut., 125: 35-41.

53. Shoba G, Joy D, Joseph T, Majeed M, Rajendran R, and Srinivas PS. (1998): Influence of piperine on the pharmacokinetics of curcumin in animals and human volunteers. Planta Med., 64: 353-356.

54. Singh J, Dubey RK, and Atal CK. (1986): Piperine-mediated inhibition of glucuronidation activity in isolated epithelial cells of the guinea-pig small intestine: evidence that piperine lowers the endogeneous UDP-glucuronic acid content. J. Pharmacol. Exp.Therap; 236: 488-493.

55. Soni KB, Kuttan R. (1992): Effects of curcumin administration on serum peroxides and cholesterol levels in human volunteers. Indian J Physiol Pharmacol.; 36(4): 273-275.

56. Sorci-Thomas M, Wilson MD, Johnson FL, Willams DL, and Rudel LL. (1989): Studies on the Expression of genes encoding apolipoprotein, B100 and B48 and the low density lipoprotein receptor in nonhuman primates. J. Biol. Chem., 264: 9039-9045.

57. Soudamini KK, Unnikrishnan MC, Soni KB, and Kuttan R. (1992): Inhibition of lipid peroxidation and cholesterol levels in mice by curcumin. Indian J. Physiol Pharmacol., 36: 239-243.

58. Sreejayan and Rao MN. (1994): Curcuminoids as potent inhibitors of lipid peroxidation. J Pharm. Pharmacol.; 46(12): 1013-1016.

59. Sreejayan and Rao MN. (1996): Free radical scavenging activity of curcuminoids. Arzneimittelforschung; 46: 169-171.

60. Srinivasan K, and Sambaiah K. (1991): The effect of spices on cholesterol 7 alphahydroxylase activity and on serum and hepatic cholesterol levels in the rat. Int. J. Vitam. Nutri. Res.; 61(4): 364-369.

61. Suryanarayana P, Krishnaswamy K, and Reddy GB. (2003): Effects of curcumin on galactose induced cataractogenesis in rats. Mol. Vis.; 9: 223-230.

62. Steel RG, and Torrie JH. (1960): Principles and procedures of statistics. McGraw Hill, Book company, Inc., New York..

63. Tebib K, Pierre B, and Jean-Max R. (1994) : Dietary grape seed Tannin effect lipoproteins, lipoprotein lipase and tissue lipids in rats fed Hypercholesterolemic diets. J. Nut., 124: 2451-2457.

64. Wang S, Chen B, and Sun C. (2000): Regulation effect of curcumin on blood lipids and antioxidation in hyperlipidemia rats. Wei. Sheng. Yan. Jiu.; 29: 240-242.

65. W. H. O. (2002): Evaluation of certain food additives and contaminants. World Health Organ Tech Rep Ser. 909: I-X, 1171 
تاثير نبات الكركوم والكركومين وخليط من الكركومين مع البييرين... على صوره ليبيدات الام في فئران التجارب البيضاء والمصابه بارتفاع ليبيدات الام الئرين

\section{غادة زغلول عباس سليمان}

زميل كيمياء حيوية قسم الكيمياء الحيوية - المعهد القومي للتغذية -ألقاهره

الكركومين ماده صفراء اللون يتم الحصول عليه من ريزومات الكركم وهو من المواد

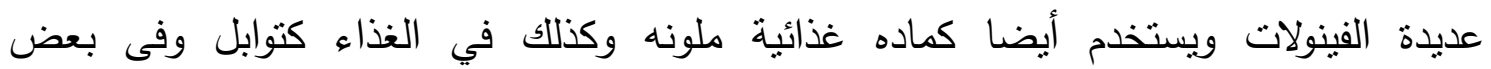

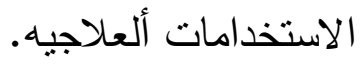
ويهدف هذا العمل لتقييم تاثير الكركوم والكركومين وخليط من الكركومين مع البييرين... على صوره ليبيدات في بلازما الفئران الطبيعية والمصابه بارتفاع اللبيدات وقد تم استخدام 270 فار قسمت إلى 27 مجموعه مج 1 ، 1 مجموعه ضابطه

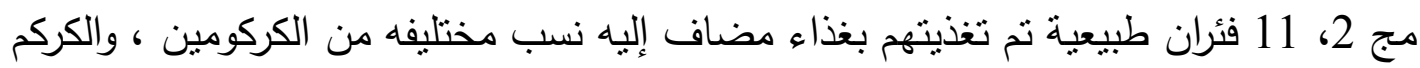

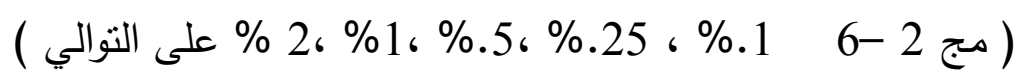

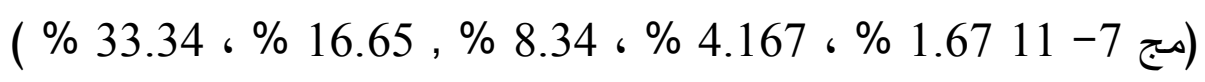

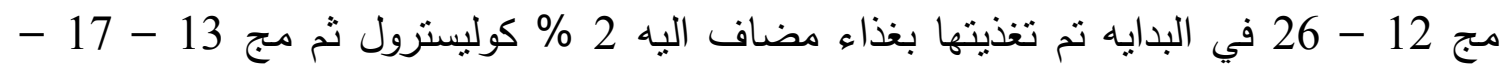
21- 25 - تم تغذيتها بغذاء مضاف اليه نسب مختلفه من الكركم والكركمين

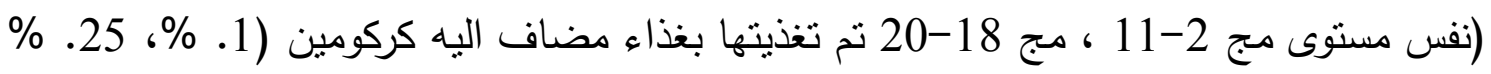

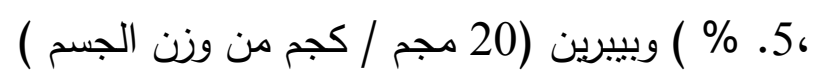
مج 12 تم ذبحها في البدايه مج 26 تم تغذيتها بغذاء طبيعي تم قياس صوره الليبيدات الجلسريدات الثناثية والفسفوليييدات " البلازما والأعضاء مثل القلب والكبد ولوحظ ارتفاع الكوليسترول (الكلى ) ، اللييوبروتينات ذات الكثافه المنخفضة ، الليبوبروتينات ذات

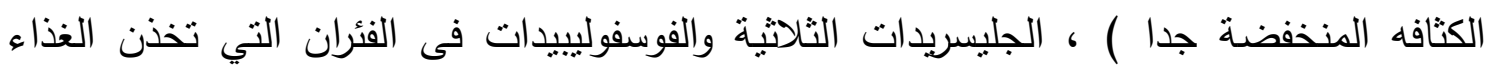
المضاف اليه كوليسترول. فبما لوحظ انخفاض اللييوبروتينات ذات الكثافه العاليه. 


\section{GHADA, Z. A. Soliman}

ان الكركم والكركومين لله تاثثر مخضض (منثط) للكوليسترول على كل من الفئران السليمه

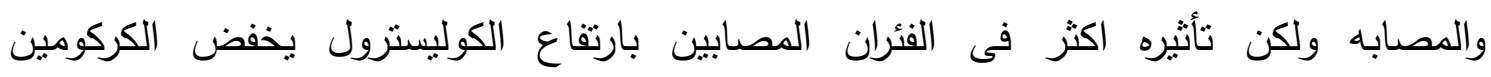

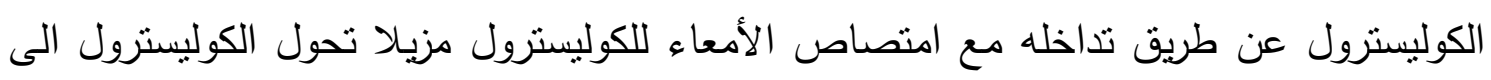

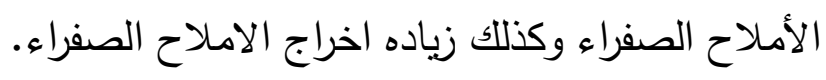

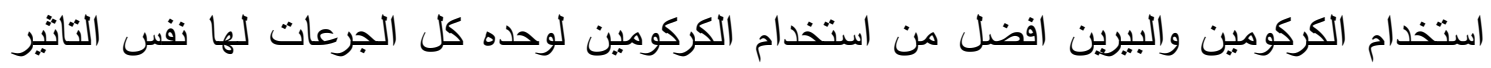
تقريبا ولكن استخدام 5.\% افضل من استخدام 2\% لوحظ ارتفاع الكوليسترول، الجليسريدات

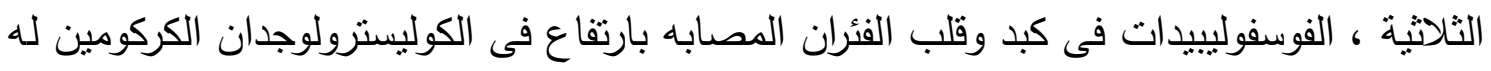
تاثثر مضاد (مخفض) لهذه النأثثرات يضاف البيرين الى الكركومين ليحسن من فاعليته عن طريق زياده امتصاصه وتقليل التمثيل فى الكبد وهكذا يمكن القول ان استخدام الكركومين ، الكركم ، خليط من الكركومين والبيرين مفيد فى التحكم فى اراضى القلب واستخدام التركيز الأقل 5. 5. افضل من استخدام 2\% ويحسن البيرين من فاعليه الكركومين. 